\title{
Large Momentum Properties and Wilson Short Distance Expansion in Non-Perturbative Field Theory
}

\author{
D. Iagolnitzer ${ }^{1}$ and J. Magnen ${ }^{2}$ \\ ${ }^{1}$ Service de Physique Théorique ${ }^{\star}$ de Saclay, F-91191 Gif-sur-Yvette Cedex, France \\ ${ }^{2}$ Centre de Physique Théorique ${ }^{\star \star}$, Ecole Polytechnique, F-91128 Palaiseau Cedex, France
}

\begin{abstract}
Large momentum properties and Wilson-Zimmerman shortdistance expansion are established via phase-space analysis for the weakly coupled massive Gross-Neveu model in dimension 2. Methods are applicable more generally.
\end{abstract}

\section{Introduction}

The aim of this work is to show how large momentum and (related) short-distance properties can be rigorously established in non-perturbative field theory via a refined application of phase-space analysis. We consider for definiteness the massive, weakly coupled Gross-Neveu model in dimension 2 [1] whose renormalization parts are as in $\varphi_{4}^{4} 2$ - and 4-point functions, and which exists as a non-trivial, asymptotically free theory recently constructed at weak (renormalized) coupling in $[2,3]$. Methods are, however, applicable more generally.

Results in Sects. 2 and 3 apply to euclidean functions. The extension to cases when some of the variables are non-euclidean (and in fact are fixed in Minkowski energy-momentum space while others tend to infinity in euclidean space) is discussed in Sect. 4: as recalled below, this is needed e.g. for "field theory" versions of Wilson-Zimmerman short-distance expansion.

Large momentum properties of the 4-point connected, amputated function $F\left(p_{1}, \ldots, p_{4}\right)$ are established in Sect. 2. The method is based on a suitable modification of the renormalization procedure of $[2,4]$ : effective couplings of vertices involving external lines will depend on corresponding energy-momenta. The reason for this modification is explained in Sect. 2.1. It amounts to the introduction of effective couplings for composite operators.

Results include in particular

(i) "Generic" situations

\footnotetext{
* Laboratoire de l'Institut de Recherche Fondamentale du Commissariat à l'Energie Atomique $\star \star$ Laboratoire propre du Centre National de la Recherche Scientifique de la République Française
} 


\section{Theorem 1.}

$$
F\left(\tau q_{1}+r_{1}, \ldots, \tau q_{4}+r_{4}\right) \sim \frac{\operatorname{cst}\left(q_{1}, \ldots, q_{4}, r_{1}, \ldots, r_{4}\right)}{\ln \tau}
$$

as $\tau \rightarrow \infty$, whenever $q_{i}+q_{j} \neq 0, \forall(i, j), i \neq j$.

(ii) "Exceptional" situations

Let $k=p_{1}+p_{2}, z=\frac{p_{1}-p_{2}}{2}, z^{\prime}=\frac{p_{3}-p_{4}}{2}$. We give below results in cases where $k$ is fixed:

\section{Theorem 2.}

$$
F\left(k, z, z^{\prime}\right) \sim \frac{\operatorname{cst}\left(k, z^{\prime}\right)}{\ln |z|^{1-\sigma}}
$$

as $|z| \rightarrow \infty$, where $\sigma \leqq \frac{1}{2}$ depends on the channel defined by particles 1,2 ( fields $\psi$ or $\bar{\psi}$, spin and colour indices; $\sigma=\frac{1}{2}$ if these indices are the same. Otherwise, $\sigma=O(1 / \mathscr{N})$, where $\mathcal{N} \geqq 2$ is the colour number).

\section{Theorem 3.}

$$
\begin{aligned}
& F\left(k, z, z^{\prime}\right) \sim \frac{\operatorname{cst}(k)}{(\ln |z|)^{1-\sigma}\left(\ln \left|z^{\prime}\right|\right)^{\sigma}} \quad \text { if } \quad \sigma<1 / 2, \\
& F\left(k, z, z^{\prime}\right) \sim \frac{\operatorname{cst}(k) \ln \ln \left|z^{\prime}\right|}{(\ln |z|)^{1 / 2}\left(\ln \left|z^{\prime}\right|\right)^{1 / 2}} \quad \text { if } \quad \sigma=1 / 2,
\end{aligned}
$$

as $|z|,\left|z^{\prime}\right| \rightarrow \infty,|z| \geqq\left|z^{\prime}\right|$.

For $(N+2)$-point functions $F_{N+2}$, Theorem 2 is still valid, $z^{\prime}$ being a set of $N-1$ relative energy-momenta among $p_{3}, \ldots, p_{N+2}$.

In Sect. 3, the aim is to establish factorization properties in momentum space (at $k, z^{\prime}$ fixed, $z \rightarrow \infty$ ) of the set of functions $F_{N+2}$, which are (essentially) equivalent to Wilson-Zimmerman short-distance expansion [5-8]. We denote below by $\omega(k, z)$ the product $S\left(p_{1}\right) S\left(p_{2}\right)$ of 2-point functions $\left(p_{1}=\frac{k}{2}+z, p_{2}=\frac{k}{2}-z\right)$.

Theorem 4 (first order expansion).

$$
F_{N+2}\left(k, z, z^{\prime}\right)=\frac{\omega(0, z)}{\omega(k, z)} F_{4}(0, z, 0) \Lambda_{N}\left(k, z^{\prime}\right)+R_{N+2}\left(k, z, z^{\prime}\right)
$$

with

$$
\left|R_{N+2}\left(k, z, z^{\prime}\right)\right|<\frac{\operatorname{cst}\left(k, z^{\prime}\right)}{1+|z|}
$$

and

$$
\begin{aligned}
\Lambda_{N}\left(k, z^{\prime}\right) & =\delta_{2, N}+\left(1 \omega R_{N+2}\right)\left(k, z^{\prime}\right) \\
& =\lim _{\varrho \rightarrow \infty} \frac{\left(1 \omega F_{N+2}\right)^{(\varrho)}\left(k, z^{\prime}\right)}{\left(1 \omega F_{4}\right)^{(\varrho)}(0,0)},
\end{aligned}
$$


where $(1 \omega A)\left(k, z^{\prime}\right)=\int \omega(k, z) A\left(k, z, z^{\prime}\right) d z$ and $(1 \omega A)^{(\varrho)}$ is defined similarly in the theory with ultraviolet cut-off.

As a consequence of (5), (6), the behaviour of $F_{N+2}, \forall N \geqq 2$, as $z \rightarrow \infty$ or (after Fourier transformation) as its dual variable $x_{1}-x_{2}$ tends to zero, is given by $F_{4}(0, z, 0)$.

Equation (5) is established in Sect. 3.1 with remainders $R_{N+2}$ that are shown to decrease at least like $1 /|z|^{1-\varepsilon} ; \varepsilon>0$ is arbitrarily small but the result holds only for smaller and smaller values of the coupling $\lambda_{\text {ren }}$ as $\varepsilon \rightarrow 0$. In Sect. 3.2, $R_{N+2}$ is itself factorized as a main term, that does decrease at least like $1 /|z|$, and a remainder that decreases at least like $1 /|z|^{2-\varepsilon}$. This completes the proof of Theorem 4 as a byproduct. The method allows one equally to pursue the expansion to higher orders.

As already mentioned, the results of Sect. 3 apply to euclidean functions. Field theory versions of Wilson expansion would require similar results as $z \rightarrow \infty$ in euclidean space for fixed Minkowskian values of $k, z^{\prime}$. "Particle analysis" along the lines of $[9,4]$ provides results in this direction as explained in Sect. 4, where Theorem 4 is in particular established up to $s\left(\equiv-k^{2}\right)=(4 \mu)^{2}-\varepsilon$ in Minkowski space (where this $\varepsilon>0$ is independent of the previous one).

\section{Renormalization Procedure and Large Momentum Properties}

2.1. Phase-Space Expansion of Green Functions: Preliminary Background. We summarize the phase-space expansion of Green functions in euclidean space-time, following [4] which is itself based on [3] with minor modifications. A momentum cut-off is first introduced in the theory by replacing the bare propagator $\widetilde{C}(p)$ by $\widetilde{C}_{\varrho}(p)=\widetilde{C}(p) \exp -\left[M^{-2 \varrho}\left(p^{2}+m_{\varrho}^{2}\right)\right]$. The latter is itself decomposed as

$$
\widetilde{C}_{\varrho}(p)=\sum_{i=1}^{\varrho} \widetilde{C}^{(i)}(p)
$$

with

$$
\begin{aligned}
\widetilde{C}^{(1)}(p) & =\widetilde{C}(p) e^{-M^{-2}\left(p^{2}+m_{\varrho}^{2}\right)}, \\
\widetilde{C}^{(i)}(p) & =\widetilde{C}(p)\left\{e^{-M^{-2 i}\left(p^{2}+m_{Q}^{2}\right)}-e^{-M^{-2(i-1)}\left(p^{2}+m_{Q}^{2}\right)}\right\}, \quad i>1 .
\end{aligned}
$$

A corresponding decomposition of $C_{\varrho}(x)$ follows by Fourier transformation.

Euclidean phase-space expansions of e.g. connected Green functions in terms of phase-space diagrams are then obtained from cluster expansions in each momentum slice $i=1, \ldots, \varrho$ with respect to scaled lattices of size $M^{-i}$ and Mayer procedures. (Phase-space diagrams include propagator and Mayer lines between squares of the lattice in each slice $i$, and lines joining different slices which link propagator lines attached to the same interaction vertex.) The expansion would be convergent (uniformly in $\varrho$ ), at small (bare) coupling constant, if any connected subdiagram $\mathbf{D}^{(j)}$ obtained in slices $\geqq j$ from a cut at slice $j$ (and having at least one propagator line in slice $j$ ) had strictly more than 4 outgoing lines below $j$, true external lines (corresponding to external particles $1, \ldots, 4$ ) being considered as belonging to slice 1: this would yield for all diagrams exponential fall off factors 
$M^{-l_{\text {sup }}}$ allowing resummations over the indices of the momentum slices; $l_{\text {sup }}$ is the index of the highest internal line. This condition is not satisfied. The renormalization procedure amounts to a rearrangement of terms of the expansion, starting from slice $\varrho$ and then proceeding to lower and lower slices. At each stage a new expansion is obtained with new effective couplings, as we now explain at a given stage corresponding to slice $j$. Any 4-point function associated with a subdiagram $\mathbf{D}^{(j)}$ with 4 outgoing lines below $j$, can be written (in euclidean energy-momentum space) as:

$$
\widetilde{D}^{(j)}\left(k_{1}, \ldots, k_{4}\right)=\left[\prod_{v} \lambda(v)\right] \mathfrak{d}^{(j)}\left(k_{1}, \ldots, k_{4}\right),
$$

where $k_{1}, \ldots, k_{4}$ are the 4 outgoing energy-momenta $\left(k_{1}+\ldots+k_{4}=0\right)$ and, for each vertex $v$ of the subdiagram, $\lambda(v)$ is the effective coupling (obtained at that stage). One then writes:

$$
\mathscr{d}^{(j)}\left(k_{1}, \ldots, k_{4}\right)=\tilde{d}_{\text {reg }}^{(j)}\left(k_{1}, \ldots, k_{4}\right)+\mathscr{d}^{(j)}(0,0,0,0),
$$

i.e. $\widetilde{d}_{\mathrm{reg}}^{(j)}=\mathfrak{d}^{(j)}-\mathfrak{d}^{(j)}(0)$. This subtraction yields in euclidean space time gradients on outgoing lines and internal regularization factors. From the viewpoint of power counting, this is equivalent to having two more outgoing lines. On the other hand, the factor $\sum_{\mathbf{D}^{(j)}}[\Pi \lambda(v)] \mathcal{d}^{(j)}(0, \ldots, 0)=\delta \lambda_{j}$ yields a redefinition of the effective coupling constants of vertices all of whose lines belong to slices $<j$. Diagrams with 2 outgoing lines below $j$ are also treated. One is finally led to a new expansion in which:

(i) each vertex with highest line $l$ has an effective coupling $\lambda_{l}=\lambda_{\varrho}+\sum_{k=l+1}^{\varrho} \delta \lambda_{k}$, where $\lambda_{\varrho} \equiv \lambda$ is the bare coupling,

(ii) all new subdiagrams $\mathbf{D}^{(j)}$ have an equivalent of at least six outgoing legs below $j, \forall j$.

With a choice of the bare coupling $\lambda=\lambda_{\varrho}$ depending on $\varrho$, of the form:

$$
\lambda_{\varrho}=\left[-\beta_{2}(\ln M) \varrho+\beta_{3} \ln \varrho+D\right]^{-1} \quad\left(\beta_{2}<0\right)
$$

with $D$ sufficiently large (independent of $\varrho$ ), it can be checked that $\lambda_{i}$ has a similar expression (in which $\varrho$ is replaced by $i$ ), so that all $\lambda_{i}$ remain small $\left(\left|\lambda_{i}\right|<1 / D\right.$, $\forall i=1, \ldots, \varrho$ ) and the new expansion is convergent (uniformly in $\varrho$ ). Since $\lambda_{1}$ is of the order of $1 / D, \lambda_{\text {ren }}$ which is equal to $\lambda_{1}$ at first order in $\lambda_{1}$, is thus itself different from zero: non-triviality. (Similarly an adequate choice of $m_{\varrho}$ depending on $\varrho$ is made; $m_{\varrho} \rightarrow 0$ in the $\varrho \rightarrow \infty$ limit, but $m_{\text {ren }}$ and the physical mass are $>0$.)

The phase-space expansion can be directly obtained for non-amputated connected Green functions $S^{c}$, or for "semi-amputated" functions $H$ from which external bare propagators have been factored out, or for amputated functions $F$. Some "particle analysis" is used in the latter case, as done in [4], by introducing from the outset cluster expansions "of order $r$," $r>1[9,4]$. This is not needed for present purposes, at least as long as we are interested only in purely euclidean functions. It is convenient in this section to treat semi-amputated functions.

2.2. Modified Renormalization Procedure. The renormalization procedure described in Sect. 2.1 is appropriate for the construction of the model but is not 
sufficient for the study of large momentum properties. A preliminary difficulty is that some of the outgoing lines of some subdiagrams $\mathbf{D}^{j}$ may correspond to actual external variables. In this case, gradients coming from renormalization will act on the functions $e^{i \Sigma \pm p_{k} x_{k}}$ involved in the Fourier transformation and will a priori yield unwanted factors $\left|p_{k}\right|$ in bounds on momentum-space functions which will not in general be compensated by factors $M^{-j}$ coming from internal regularization factors.

The way which seems most convenient to study large momentum properties is to avoid this difficulty by modifying the renormalization procedure as follows.

Given the external energy-momenta $p_{k}, k=1, \ldots, 4$, let $i_{k} \equiv i\left(p_{k}\right)$ be the $\geqq 1$ integer such that $M^{i_{k}-1}<\left|p_{k}\right| \leqq M^{i_{k}}$ : the external line $k$ will be considered by convention to belong to slice $i_{k}$. $M$ is chosen below $\geqq 2$ and we assume e.g. (without loss of generality) in this section that

$$
i_{1} \geqq i_{2} \geqq i_{3} \geqq i_{4} \text {. }
$$

Similarly $i_{\alpha, \beta} \equiv i\left(p_{\alpha}+p_{\beta}\right), i_{\alpha, \beta, \gamma} \equiv i\left(p_{\alpha}+p_{\beta}+p_{\gamma}\right) ; i_{\alpha, \beta, \gamma}=i_{\delta}(\alpha, \beta, \gamma, \delta$ are different indices among $1, \ldots, 4)$.

For any connected subdiagram $\mathbf{D}^{(j)}$ containing $n_{\text {ext }}(\mathbf{D}) \geqq 1$ external lines with indices $\geqq j$ among its 2 or 4 outgoing lines, the subtraction will now be as follows. [There is no subtraction if there is no outgoing line below $j$. On the other hand, the rule of Sect. 2.1 is unchanged if $n_{\text {ext }}(D)=0$.]

(i) No subtraction if $n_{\mathrm{ext}}(\mathbf{D})=1$, or $n_{\mathrm{ext}}(\mathbf{D})=2$ and $j<i_{\alpha, \beta}$, where $\alpha, \beta$ are the indices of the 2 external lines in slices $\geqq j$, or $n_{\text {ext }}(\mathbf{D})=3$ and $j<i_{\alpha, \beta, \gamma}$, where $\alpha, \beta, \gamma$ are the indices of the 3 external lines in slices $\geqq j$.

In fact, energy-momentum conservation and the ultraviolet cut-off factors in the definition of the propagators $\widetilde{C}^{(l)}, l<j$, of the outgoing lines below $j$, ensure exponential fall-off factors much better than $M^{-\left(j-l_{1}\right)}$, where $l_{1}$ is the index of the highest outgoing line below $j$, which make renormalization useless.

(ii) In the other cases:

a) $n_{\text {ext }}(\mathbf{D})=2$, external momenta $p_{\alpha}, p_{\beta}, 2$ outgoing lines below $j\left(j>i_{\alpha, \beta}\right)$. From $\left[\prod_{v} \lambda(v)\right] \tilde{d}^{(j)}\left(p_{\alpha}, p_{\beta}, k_{3}, k_{4}\right)$ we now subtract $\left[\prod_{v} \lambda(v)\right] \tilde{d}^{(j)}\left(\frac{p_{\alpha}-p_{\beta}}{2},-\frac{p_{\alpha}-p_{\beta}}{2}, 0,0\right)$ (i.e. the outgoing energy-momenta $k_{3}, k_{4}$ below $j$, hence also $k=k_{1}+k_{2}$, have been fixed at zero).

We note that the effective coupling $\lambda(v)$ and the terms $\widetilde{d}^{(j)}$ differ here from those of Sect. 2.1 although we have used the same notation. In particular, the effective couplings of vertices involving external lines will now depend on their momenta.

b) $n_{\text {ext }}(\mathbf{D})=3$, external momenta $p_{1}, p_{2}, p_{3}, 1$ outgoing line below $j$. Subtracted term:

$$
\left[\prod_{v} \lambda(v)\right] \mathcal{d}^{(j)}\left(p_{1}, p_{2},-\left(p_{1}+p_{2}\right), 0\right)
$$

The sum over diagrams $\mathbf{D}^{(j)}$ defines counterterms $\delta m_{j}, \delta \xi_{j}, \delta \lambda_{j}$ which are the usual ones and new counterterms $\delta \lambda_{j}\left(p_{\alpha}, p_{\beta}\right), \delta \lambda_{j}\left(p_{\alpha}, p_{\beta}, p_{\gamma}\right)$ in cases (ii). They also depend in cases (ii) of the channel defined by the external lines. Effective coupling constants of vertices with 4 lines will be the same as in Sect. 2.1 except for (at most 
4) vertices involving external lines: see Sect. 2.3. The new procedure yields gradients acting only on outgoing lines below $j$, and still yields internal regularization factors. Exponential factors in $M^{-l_{\text {sup }}}$ are, however, replaced by $M^{-\left(l_{\text {sup }}-l_{\text {nff }}\right)}$, where $l_{\text {sup }}$ is as in Sect. 2.1 the index of the highest internal line of the diagram and $l_{\text {inf }}$ is the lowest index of all internal and external lines $\left(l_{\text {inf }}\right.$ was always equal to 1 in Sect. 2.1, since external lines were by convention in slice 1, and was thus omitted). All summations over slice indices are thus again controlled by those factors as before, except the summation over $l_{\text {inf }}$ from slice 1 to $i_{4}$ at most. This summation, which would a priori give at most a factor $i_{4} \sim \ln \left|p_{4}\right|$ in bounds on connected semi-amputated or amputated functions, will be made in Sect. 2.4 by taking into account the behaviour of effective couplings of vertices involving external lines, so that better results will be obtained.

2.3. Effective Couplings. Given any vertex, let $i$ denote the highest slice of the 4 lines attached to it. The effective coupling is $\lambda_{i}$ (as defined in Sect. 2.1) if the vertex involves no external line or if at least one of the two highest lines is an internal one. In remaining cases:

(i) If the two highest lines are external lines of indices $\alpha, \beta$ and if the third one is an internal line of index $j$, then the effective coupling is:

$$
\begin{array}{ll}
\lambda_{j}\left(p_{\alpha}, p_{\beta}\right) \equiv \lambda_{i_{\alpha}}+\sum_{k=j+1}^{i_{\beta}} \delta \lambda_{k}\left(p_{\alpha}, p_{\beta}\right) & \text { if } j \geqq i_{\alpha, \beta}, \\
\lambda_{i_{\alpha, \beta}}\left(p_{\alpha}, p_{\beta}\right) & \text { if } j<i_{\alpha, \beta} .
\end{array}
$$

(ii) If the three highest lines are external lines of indices $i_{\alpha}, i_{\beta}, i_{\gamma}\left(i_{\alpha} \geqq i_{\beta} \geqq i_{\gamma}\right)$ and the fourth line is of index $j$, the effective coupling is:

a) $(\alpha, \beta, \gamma)=(1,2,3)$

$$
\begin{array}{ll}
\lambda_{j}\left(p_{\alpha}, p_{\beta}, p_{\gamma}\right) \equiv \lambda_{i_{3}}\left(p_{1}, p_{2}\right)+\sum_{k=j+1}^{i_{3}} \delta \lambda_{k}\left(p_{1}, p_{2}, p_{3}\right) & \text { if } j \geqq i_{4}, \\
\lambda_{i_{4}}\left(p_{1}, p_{2}, p_{3}\right) & \text { if } j<i_{4} .
\end{array}
$$

This case includes the vertex with 4 external lines with effective coupling $\lambda_{i_{4}}\left(p_{1}, p_{2}, p_{3}\right)$.

b) $(\alpha, \beta, \gamma) \neq(1,2,3)$

$$
\begin{array}{rll}
\lambda_{i_{\gamma}}\left(p_{\alpha}, p_{\beta}\right) & \text { if } & i_{\gamma} \geqq i_{\alpha, \beta}, \\
\lambda_{i_{\alpha, \beta}}\left(p_{\alpha}, p_{\beta}\right) & \text { if } & i_{\gamma}<i_{\alpha, \beta} .
\end{array}
$$

Evaluation of $\delta \lambda_{j}\left(p_{\alpha}, p_{\beta}\right)$ and of $\lambda_{j}\left(p_{\alpha}, p_{\beta}\right)$. We use below expansions of the phasespace diagrams contributing to $\delta \lambda_{j}\left(p_{\alpha}, p_{\beta}\right)$ as terms of second order in the effective couplings and remainders. The sum of the latter is bounded by $\operatorname{cst} \lambda_{j}\left(p_{\alpha}, p_{\beta}\right) \lambda_{j}^{2}$. [Convergence is ensured as usual by the smallness of the effective couplings and there are at least 3 couplings one of which is bounded by $\lambda_{j}\left(p_{\alpha}, p_{\beta}\right)$ and others by $\lambda_{j}$.] Terms with two couplings, i.e. two vertices $v_{1}, v_{2}$ have two internal (propagator) lines in slices $j, j^{\prime}$ respectively, $j^{\prime} \geqq j$, running between $v_{1}, v_{2}$ and two more outgoing lines at each vertex, including the two external lines $\alpha, \beta$ which may or not be attached to the same vertex: see Fig. 1. 

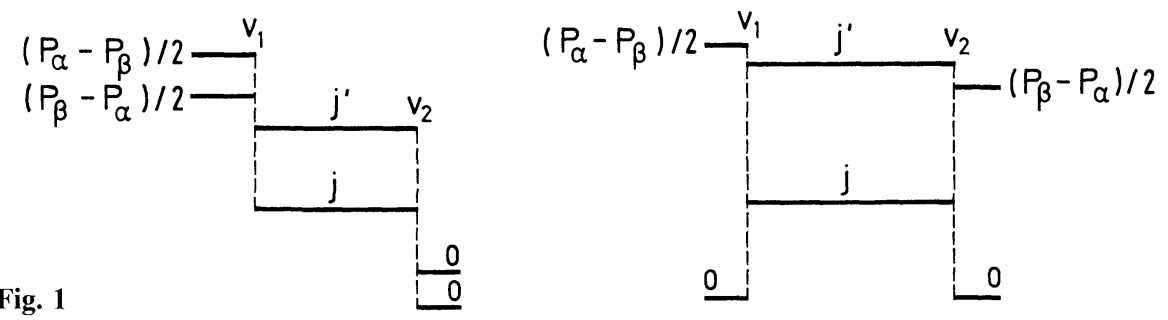

Correspondingly:

$$
\begin{aligned}
\delta \lambda_{j}\left(p_{\alpha}, p_{\beta}\right)= & \sum_{j^{\prime} \geqq j} \mathcal{d}\left(j, j^{\prime},(\alpha, \beta)\right) \times \lambda_{j^{\prime}} \times\left\{\begin{array}{lll}
\lambda_{j^{\prime}} & \text { if } j^{\prime} \geqq i_{\beta} \\
\lambda_{j^{\prime}}\left(p_{\alpha}, p_{\beta}\right) & \text { if } j^{\prime}<i_{\beta}
\end{array}\right\} \\
& +\sum_{j^{\prime} \geqq j} \widetilde{d}\left(j, j^{\prime} ; \alpha ; \beta ; \frac{p_{\alpha}-p_{\beta}}{2}\right)\left\{\begin{array}{lll}
\lambda_{j^{\prime}} & \text { if } & j^{\prime} \geqq i_{\alpha} \\
\lambda_{i_{\alpha}} & \text { if } & j^{\prime}<i_{\alpha}
\end{array}\right\} \times\left\{\begin{array}{lll}
\lambda_{j^{\prime}} & \text { if } j^{\prime} \geqq i_{\beta} \\
\lambda_{i_{\beta}} & \text { if } \quad j^{\prime}<i_{\beta}
\end{array}\right\} \\
& + \text { terms with } 3 \text { couplings or more. }
\end{aligned}
$$

In (17), $\widetilde{d}\left(j, j^{\prime},(\alpha, \beta)\right)=\operatorname{cst}(\alpha, \beta) \int \widetilde{C}^{(j)}(k) \widetilde{C}^{\left(j^{\prime}\right)}(-k) d k: \alpha, \beta$ attached to the same vertex, external momentum of the channel $(\alpha, \beta)$ fixed at zero. It depends on the channel determined by $(\alpha, \beta)$ (fields $\Psi$ or $\bar{\Psi}$, spin and colour indices). On the other hand $\tilde{d}\left(j, j^{\prime}, \alpha ; \beta ; q\right)=\operatorname{cst}(\alpha, \beta) \int \widetilde{C}^{(j)}(k) \times \widetilde{C}^{\left(j^{\prime}\right)}(-k+q) d k: \alpha, \beta$ attached to $v_{1}$ and $v_{2}$ respectively, external momentum of the channel $\alpha, . \rightarrow \beta$. fixed at $q=\frac{p_{\alpha}-p_{\beta}}{2}+0$ $=\frac{p_{\alpha}-\frac{p_{\beta}}{2}}{2}$. It also depends on the characteristics of $\alpha, \beta$. (Fields $\psi$ or $\bar{\psi}$, spin and colour indices. There is possibly a summation over the characteristics of remaining lines.)

The following remarks will be useful:

$$
\begin{gathered}
\left|\tilde{d}\left(j, j^{\prime},(\alpha, \beta)\right)\right|<\operatorname{cst} M^{-\left(j^{\prime}-j\right)}, \\
\sum_{j^{\prime} \geqq j} \tilde{d}\left(j, j^{\prime},(\alpha, \beta)\right)=\sigma \beta_{2} \ln M,
\end{gathered}
$$

where $\sigma \equiv \sigma(\alpha, \beta)$ depends on the channel $(\alpha, \beta)$.

On the other hand, recalling that $i_{\beta} \geqq j>i_{\alpha \beta}, j^{\prime} \geqq j$ and using energy-momentum conservation arguments,

$$
\left|\widetilde{d}\left(j, j^{\prime} ; \alpha ; \beta, \frac{p_{\alpha}-p_{\beta}}{2}\right)\right| \leqq \operatorname{cst} M^{-\left(j^{\prime}-j\right) / 2} M^{-\left(i_{\alpha}-j\right) / 2} .
$$

In formula (17), we now express $\lambda_{j^{\prime}}$ and $\lambda_{j^{\prime}}\left(p_{\alpha}, p_{\beta}\right)$ as $\lambda_{j}-\sum_{k=j+1}^{j^{\prime}} \delta \lambda_{k}$ and $\lambda_{j}\left(p_{\alpha}, p_{\beta}\right)$ - $\sum_{k=j+1}^{j^{\prime}} \delta \lambda_{k}\left(p_{\alpha}, p_{\beta}\right)$ respectively. The sums over $k$ are controlled by the exponential factors $M^{-\left(j^{\prime}-j\right)}$ or $M^{-\left(j^{\prime}-j\right) / 2}$ in (18), (19) so that we can replace in (17) $\lambda_{j^{\prime}}$ and $\lambda_{j^{\prime}}\left(p_{\alpha}, p_{\beta}\right)$ by $\lambda_{j}, \lambda_{j}\left(p_{\alpha}, p_{\beta}\right)$ up to third order contributions. Hence:

$\delta \lambda_{j}\left(p_{\alpha}, p_{\beta}\right)=\lambda_{j}\left(p_{\alpha}, p_{\beta}\right) \lambda_{j} \sigma \beta_{2} \ln M+\lambda_{j}^{2} O\left(M^{-\left(i_{\beta}-j\right) / 2}\right)+$ higher order terms 
and in turn:

$$
\begin{aligned}
\lambda_{j-1}\left(p_{\alpha}, p_{\beta}\right) & =\lambda_{j}\left(p_{\alpha}, p_{\beta}\right)\left[1+\lambda_{j} \sigma \beta_{2} \ln M+O\left(\lambda_{j}^{2}\right)\right]+\lambda_{j}^{2} O\left(M^{-\left(i_{\beta}-j\right) / 2}\right) \\
& =\lambda_{i_{\beta}} \prod_{k=j+1}^{i_{\beta}}\left[1+\lambda_{k} \sigma \beta_{2} \ln M+O\left(\lambda_{k}^{2}\right)\right]\left[1+O\left(\lambda_{i_{\beta}}\right)\right] \\
& =\lambda_{i_{\beta}}\left(\frac{i_{\beta}}{j}\right)^{\sigma}(1+O(1 / j))\left[1+O\left(\lambda_{i_{\beta}}\right)\right] .
\end{aligned}
$$

Hence $\lambda_{j}\left(p_{\alpha}, p_{\beta}\right)$ behaves like $\frac{1}{i_{\beta}^{1-\sigma}} \frac{1}{j^{\sigma}}$ at large $i_{\beta}$ and $j, j \leqq i_{\beta}$.

Evaluation of $\delta \lambda_{j}\left(p_{1}, p_{2}, p_{3}\right), i_{3} \geqq j>i_{4}$. Leading contributions to $\delta \lambda_{j}\left(p_{1}, p_{2}, p_{3}\right)$ are again diagrams with two vertices $v_{1}, v_{2}$, and two internal propagator lines in slices $j, j^{\prime}, j^{\prime} \geqq j$, running between $v_{1}, v_{2}$. If external lines 1,2 are attached to $v_{1}$, and 3 (as also a further outgoing line) attached to $v_{2}$, energy-momenta of these 4 outgoing lines are fixed to $p_{1}, p_{2},-\left(p_{1}+p_{2}\right)$ and zero respectively. Couplings at $v_{1}, v_{2}$ are $\lambda_{i_{1,2}}\left(p_{1}, p_{2}\right)$ and $\lambda_{i_{3}}$. Summation over $j, j^{\prime}$ is controlled by exponential factors $M^{-\left(j^{\prime}-j\right)}$ and (by energy-momentum conservation arguments) $M^{-\left|i_{1,2}-j\right|}$. Other terms with 2 couplings have the external lines 1,3 or 2,3 attached to $v_{1}$, while 2 or 1 is attached to $v_{2}$. Energy-momenta of these lines are fixed at $p_{\alpha},-\left(p_{1}+p_{2}\right)$ and $p_{\beta},(\alpha, \beta)=(1,2)$ or $(2,1)$. Couplings are $\lambda_{i_{1}}$ and $\lambda_{i_{2}}$. Taking also into account uniform convergence properties of higher order terms, this gives finally:

$$
\left|\sum_{j=i_{4}+1}^{i_{3}} \delta \lambda_{j}\left(p_{1}, p_{2}, p_{3}\right)\right| \leqq O(1) \frac{1}{i_{1}^{1-\sigma}} \frac{1}{i_{1,2}^{\sigma}} \frac{1}{i_{3}} .
$$

2.4. Bounds on $H\left(p_{1}, \ldots, p_{4}\right)$ or $F\left(p_{1}, \ldots, p_{4}\right)$.

\section{Theorem 5.}

$$
\left|H\left(p_{1}, \ldots, p_{4}\right)\right|<O(1) \frac{1}{i_{1}^{1-\sigma(1,2)}} \frac{1}{i_{3}^{\sigma(1,2)}} \operatorname{Sup} \log \left(i_{4} / i_{\alpha, \beta}\right)
$$

where the Sup runs over values of $\alpha, \beta$ such that $\sigma(\alpha, \beta)=\frac{1}{2}, i_{\alpha, \beta}<i_{4}$.

Remark. The last factor can always be removed if $i_{4}<i_{3}-1$. Moreover if $\sigma(1,2)$ $<1 / 2$, it can be removed if $i_{4}<i_{1}-2$.

Proof. Diagrams contributing to $H$ are divided into the following classes:

(i) the trivial diagram with one vertex (whose effective coupling has been given in Sect. 2.3).

(ii) diagrams including 2 vertices $v^{\prime}, v^{\prime \prime}$ each of which involving 2 external lines $(1, \beta)$ and $(\gamma, \delta)$ respectively, and whose highest internal line $l_{\text {sup }}$ is smaller than $i_{4}$. If $l^{\prime}, l^{\prime \prime}$ denote the highest internal lines attached to $v^{\prime}$ and $v^{\prime \prime}$ respectively, effective couplings behave like $\frac{1}{i_{1}^{1-\sigma(1, \beta)}} \frac{1}{l^{\prime \sigma(1, \beta)}}$ and $\frac{1}{i_{\gamma}^{1-\sigma(1, \beta)}} \frac{1}{l^{\prime \prime \sigma(1, \beta)}}$. Given $l_{\text {inf }}$, the sum over possible slices is controlled by the exponential fall-off factor $M^{-\left(l_{\text {sup }}-l_{\text {nff }}\right)}$. The sum over $l_{\text {inf }}$ below $i(1, \beta)=i\left(p_{1}+p_{\beta}\right)$ is also controlled by exponential fall-off factors in view of energy-momentum conservation and the ultraviolet cut-off factors in the 
propagators $\widetilde{C}^{(.)}$of internal lines attached to $v^{\prime}$ or $v^{\prime \prime}$. The sum over $l_{\text {inf }}$ between $i(1, \beta)$ and $i_{4}$ is made by using:

$$
\sum_{l_{\mathrm{nf}}=i(1, \beta)}^{i_{4}} \frac{1}{l_{\mathrm{inf}}^{2 \sigma(1, \beta)}}=\left\{\begin{array}{lll}
i_{4}^{1-2 \sigma}-i(1, \beta)^{1-2 \sigma} & \text { if } & \sigma \neq \frac{1}{2} \\
\ln i_{4}-\ln i(1, \beta) & \text { if } & \sigma=\frac{1}{2}
\end{array} .\right.
$$

(iii) all remaining diagrams can also be treated by taking into account the behaviour of couplings involving external lines and exponential factors analogous to the above. Their sum yields better bounds than those arising from cases (i), (ii) which are thus at the origin of the results of Theorem 5.

Proof of Theorems 1, 2, 3 of Sect. 1. In the case of Theorem $1, i_{1}, \ldots, i_{4}$ as also $i(1, \beta), \beta=2,3,4$ are all proportional to $\ln \tau$. The decrease of $H$ at least in $1 / \ln \tau$ follows. The fact that this is the actual behavior of $H$ comes from the evaluation of the trivial diagram and of diagrams with two vertices. Other terms are smaller.

In the case of Theorem 2: $i_{1} \sim i_{2} \rightarrow \infty, i(1,2), i_{3}, i_{4}$ remaining small. As a consequence:

$$
\lambda_{i_{4}}\left(p_{1}, p_{2}, p_{3}\right) \sim \frac{O(1) \operatorname{cst}\left(p_{3}\right)}{i_{1}^{1-\sigma(1,2)}}, \quad(\text { two vertices diagrams }) \sim \frac{\operatorname{cst}\left(p_{3}, p_{4}\right)}{i_{1}^{1-\sigma(1,2)}} .
$$

In the case of Theorem 3 , only $i_{1,2}$ is small. Hence the behaviour is governed by $\sigma(1,2)$.

Remarks. 1) The same methods yield without difficulty the analogue of Theorem 2 on $(N+2)$-point functions for $k, z^{\prime}$ fixed, $z \rightarrow \infty$, where $z^{\prime}$ is a set of $N-1$ relative energy-momenta among $p_{3}, \ldots, p_{N+2}$.

In the proof of Theorem 2 and of this result, the external lines 3, 4 or $3,4, \ldots, N+2$ can from the outset be considered to belong to slice 1 .

2) All results apply in a similar way for the (connected) amputated functions $F$ in view of the relations:

$$
\omega_{b}(k, z) H\left(k, z, z^{\prime}\right) \omega_{b}\left(k, z^{\prime}\right)=\omega(k, z) F\left(k, z, z^{\prime}\right) \omega\left(k, z^{\prime}\right),
$$

where $\omega$ and $\omega_{b}$ are products of 2-point functions and of bare propagators respectively, and of the fact that $\frac{\omega(k, z)}{\omega_{b}(k, z)} \rightarrow 1$ as $z \rightarrow \infty$ (see e.g. [4]).

Results are alternatively obtained directly on the functions $F$ if (as in [4]) some "particle analysis" is made at the beginning in order to get adequate expansions of these functions.

\section{Wilson-Zimmerman Short-Distance Expansion}

We consider in this section $(N+2)$-point (connected, "semi-amputated") functions $H_{N+2}\left(p_{1}, \ldots, p_{N+2}\right)$ with $k=p_{1}+p_{2}$ and $z^{\prime}$ fixed, $z \rightarrow \infty\left(z=\left(p_{1}-p_{2}\right) / 2\right)$. We leave below $N$ implicit unless otherwise stated and express $H$ in terms of $k, z, z^{\prime}$. By convention, external lines $3, \ldots, N+2$ are considered to belong to slice 1 and lines 1,2 to slice $i(z)$. Thus for $j<i(z), \lambda_{j}\left(p_{1}, p_{2}\right)$ depends only on $z$ and will be denoted $\lambda_{j}(z)$. For $j \geqq i(z)$ by definition $\lambda_{j}(z) \equiv \lambda_{j}$.

3.1. First Order ("up to $\varepsilon$ "). Results in this section include some restrictions (decrease of relevant functions in $1 /|z|^{1-\varepsilon}, \varepsilon>0$, for couplings $\lambda_{\text {ren }}<\lambda_{0} \varepsilon$ ). Better 
results (decrease in $1 /|z|$ for couplings $\lambda_{\text {ren }}<\lambda_{0}$ ) follow from the further analysis (to next order) carried out in Sect. 3.2.

Lemma 1. $\exists \lambda_{0}>0$ such that $\forall \lambda_{\text {ren }}<\lambda_{0}, H$ can be written in the form:

$$
H\left(k, z, z^{\prime}\right)=\sum_{j \geqq 1} \lambda_{j}(z) H^{(j)}\left(k, z^{\prime}\right)+H^{\prime}\left(k, z, z^{\prime}\right)
$$

where, for any $\varepsilon>0(\varepsilon<1)$ :

$$
\begin{aligned}
\left|H^{(j)}\left(k, z^{\prime}\right)\right| & <O(1) M^{-(1-\varepsilon) j}, \\
\left|H^{\prime}\left(k, z, z^{\prime}\right)\right| & <O(1) \lambda_{\mathrm{ren}}^{2} M^{-(1-\varepsilon) i(z)},
\end{aligned}
$$

$\forall \lambda_{\text {ren }}$ such that $\lambda_{\text {ren }}<O(1) \lambda_{0} \varepsilon$.

Proof. The decomposition (26) is obtained by regrouping diagrams contributing to $H$ into the following subsets:

(i) diagrams in which the external lines 1,2 are not attached to the same vertex. The sum of these diagrams is $H^{\prime}$. In view of renormalization and energymomentum conservation (applied at the vertices involving the external lines 1,2), factors $M^{-i(z)}$ are obtained for all diagrams. A part $M^{-\varepsilon i(z)}$ is kept for internal resummations: it is sufficient for that purpose, but convergence is established only for $\lambda_{\text {ren }}<O(1) \lambda_{0} \varepsilon$. A factor $\lambda_{\text {ren }}^{2}$ can be on the other hand included because all diagrams have at least two vertices. (The factor $M^{-h(u) / 6}$ obtained for each vertex, where $h(u)$ is the difference of the extremal indices of lines attached to that vertex is replaced by $M^{-\varepsilon h(u) / 6}$ : see [4].)

(ii) diagrams in which external lines 1,2 are attached to the same vertex. Let us call $j$ the highest index of the two remaining lines attached to that vertex.

For each $j$, the term $\lambda_{j}(z) H^{(j)}(k, z)$ is obtained after factorizing out the effective coupling $\lambda_{j}(\mathrm{z})$ of the vertex involving 1,2 . (The trivial vertex is included in the term $j=1$.) The bound (27) follows again from factors $M^{-j}$ (the index of the highest internal line of contributing diagrams for each $j$ being $\geqq j$ ).

Lemma 2. There exist (unique) functions $R^{(1)}\left(k, z, z^{\prime}\right)$ and $\Lambda^{(1)}\left(k, z^{\prime}\right)$ (independent of z) such that:

$$
H\left(k, z, z^{\prime}\right)=\lambda_{1}(z) \Lambda^{(1)}\left(k, z^{\prime}\right)+R^{(1)}\left(k, z, z^{\prime}\right)
$$

with

$$
\left|R^{(1)}\left(k, z, z^{\prime}\right)\right|<O(1) \frac{\lambda_{\text {ren }}}{(1+|z|)^{1-\varepsilon}}
$$

for $\lambda_{\text {ren }}<O(1) \lambda_{0} \varepsilon$.

Remark. The uniqueness of the functions $\Lambda^{(1)}, R^{(1)}$ (at $\varrho$ infinite) is a consequence of the bound (30) on $R^{(1)}$ and of the fact that $\lambda_{1}(z)$ has a lower decrease [in $\left.1 /\left(\ln |z|^{1-\sigma}\right)\right]$ as $z \rightarrow \infty$.

Proof. We start from (26) and use the relations:

$$
\begin{gathered}
\lambda_{j}(z)=\lambda_{1}(z)+\sum_{n=2}^{j} \delta \lambda_{n}(z), \\
\delta \lambda_{n}(z)=L_{n}(z)+\sum_{l \geqq n} \lambda_{l}(z) F_{n}^{l} .
\end{gathered}
$$


In (32), $\delta \lambda_{n}(z)$ which is the sum $\sum \mathbf{D}^{(n)}(z,-z, 0,0)$ has been separated into the contribution $L_{n}$ corresponding to diagrams in which $z$ and $-z$ are attached to two different vertices, and remaining ones, that have been again separated according to the highest index $l$ of the two lines attached to the vertex involving $z,-z$. We recall that in all cases $n$ is the index of the lowest internal line of the diagrams. $F_{n}^{l}$ depends on $n, l$ but not on the momenta $k, z, z^{\prime}$.

Equations (26), (31), (32) yield (29) with:

$$
\begin{gathered}
\Lambda^{(1)}\left(k, z^{\prime}\right)=\sum H^{(j)}\left(k, z^{\prime}\right)\left[1+F_{n}^{l}+F_{n}^{l_{1}} F_{n_{1}}^{l}+\ldots+F_{n}^{l_{1}} F_{n_{1}}^{l_{2}} \ldots F_{n_{v}}^{l_{v+1}} F_{n_{v+1}}^{l}+\ldots\right], \\
R^{(1)}\left(k, z, z^{\prime}\right)=H^{\prime}\left(k, z, z^{\prime}\right)+\sum H^{(j)}\left(k, z^{\prime}\right)\left[1+F_{n}^{l}+F_{n}^{l_{1}} F_{n_{1}}^{l}+\ldots\right] L_{n^{\prime}}(z)
\end{gathered}
$$

where summations run over (integer) values of $j, n, n_{1}, \ldots, n_{v+1}, l, l_{1}, \ldots, l_{v+1}, n^{\prime}$ such that: $j \geqq 1,2 \leqq n \leqq j, l \geqq n$ for the term $F_{n}^{l}$ or more generally, $l_{1} \geqq n, 2 \leqq n_{1} \leqq l_{1}$, $l_{2} \geqq n_{1}, \ldots, 2 \leqq n_{r} \leqq l_{r}, l_{r+1} \geqq n_{r}, \ldots, l \geqq n_{v+1}, 2 \leqq n^{\prime} \leqq l$, and all possible integer values of $v$. The convergence of the right hand side of (33), (34) and the bound (30) on $R^{(1)}$ can be established as explained below from the following bounds on $F_{n}^{l}$ and $L_{n}$, obtained by the same arguments as (27), (28) for $\lambda_{\text {ren }}<O(1) \lambda_{0} \varepsilon$ :

$$
\begin{aligned}
\left|F_{n}^{l}\right| & <O(1) \lambda_{\mathrm{ren}} M^{-(1-\varepsilon)(l-n)}, \\
\left|L_{n}(z)\right| & <O(1) \lambda_{\mathrm{ren}}^{2} M^{-(1-\varepsilon)(i(z)-n)}, \quad i(z)>n, \\
\left|L_{n}(z)\right| & <O(1) \lambda_{\mathrm{ren}}^{2}, \quad n \geqq i(z) .
\end{aligned}
$$

a) Convergence of (33) and (34). We first state:

\section{Lemma 3.}

$$
\sum_{j, n, l_{1}, \ldots, n_{v+1}}\left|H^{(j)} F_{n}^{l_{1}} \ldots F_{n_{v+1}}^{l}\right|<O(1) C_{M}^{v} \lambda_{\text {ren }}^{v} M^{-(l / 2)(1-\varepsilon)}
$$

for $\lambda_{\text {ren }}<O(1) \lambda_{0} \varepsilon$.

Proof. In (38), the sum in the right-hand side runs over all values of $j, n, \ldots, l_{v+1}$, $n_{v+1}$, but not $l$, subject to the constraints already mentioned $\left(n_{v+1} \leqq l\right.$, $\left.l_{v+1} \geqq n_{v+1}, \ldots\right)$. The bound (38) is proved by induction on $v$.

From Lemma 3 , by summation over $l$, each individual term in the right-hand side of (33) satisfies itself bounds in $O(1)\left(C_{M} \lambda_{\text {ren }}\right)^{v}$, which yield in turn convergence of the series (33), (34) for $\lambda_{\text {ren }}$ small enough.

b) Results on $R^{(1)}$. For each $v$ and all possible values of $j, n, l_{1}, \ldots, l, n^{\prime}$, satisfying the constraints that have been indicated, a uniform factor such as $M^{-(1-2 \varepsilon) i(z)}$ can first be extracted (before summations) from the factors $M^{-(1-\varepsilon)(\cdot)}$ involved in the bounds on $H^{(j)}, F_{n}^{l_{1}}, \ldots$ Remaining factors $M^{-\varepsilon(\cdot)}$ will be used for resummation by the same arguments as in paragraph a). The new constant $C_{M}(\varepsilon)$, now linked to $\sum_{l} M^{-\varepsilon l / 2}$, behaves like $O(1) / \varepsilon$ at small $\varepsilon$, but desired results still hold (with suitable choices of $\lambda_{0}, \varepsilon$ ).

This ends the proof of Lemma 2 . 
We can now prove:

Theorem 4'. $\exists \lambda_{0}>0$ such that the factorization properties (5), (7) hold for $\lambda_{\text {ren }}<\lambda_{0}$ and such that, $\forall \varepsilon>0(\varepsilon<1)$ :

$$
\left|R_{N+2}\left(k, z, z^{\prime}\right)\right|<O(1) \frac{\lambda_{\text {ren }}}{(1+|z|)^{1-\varepsilon}}
$$

for $\lambda_{\text {ren }}<O(1) \lambda_{0} \varepsilon$.

Proof.

a) Apart from Eqs. (7), ( $\left.7^{\prime}\right)$, which require a more detailed analysis given in paragraph b), Theorem $4^{\prime}$ is a direct consequence of Lemma 2, of the relation (25) and of known informations on the behaviour of $\omega(k, z), \omega_{b}(k, z)$ at large $z$ (see e.g. [4]).

Equation (29), considered at $N=2, k=z^{\prime}=0$ yields:

$$
\lambda_{1}(z)=\left[H_{4}(0, z, 0)-R_{4}^{(1)}(0, z, 0)\right] / \Lambda_{2}^{(1)}(0,0),
$$

and (29) can thus be rewritten as:

$$
\begin{aligned}
H_{N+2}\left(k, z, z^{\prime}\right)= & H_{4}(0, z, 0) \frac{\Lambda_{N+2}^{(1)}\left(k, z^{\prime}\right)}{\Lambda_{2}^{(1)}(0,0)} \\
& +R_{N+2}^{(1)}\left(k, z, z^{\prime}\right)-\frac{R_{4}^{(1)}(0, z, 0)}{\Lambda_{2}^{(1)}(0,0)} \Lambda_{N}^{(1)}\left(k, z^{\prime}\right) .
\end{aligned}
$$

From (25) [applied to $H_{N+2}\left(k, z, z^{\prime}\right)$ and $H_{4}(0, z, 0)$ ], one obtains in turn Eq. (5) with:

$$
\begin{aligned}
& \Lambda_{N}\left(k, z^{\prime}\right)=\frac{\omega(0,0)}{\omega_{b}(0,0)} \frac{\Lambda_{N}^{(1)}\left(k, z^{\prime}\right)}{\Lambda_{2}^{(1)}(0,0)} \frac{\omega_{b}\left(k, z^{\prime}\right)}{\omega\left(k, z^{\prime}\right)} \\
& R_{N+2}\left(k, z, z^{\prime}\right)= \frac{\omega_{b}(k, z)}{\omega(k, z)}\left[R^{(1)}\left(k, z, z^{\prime}\right)-R^{(1)}(0, z, 0) \frac{\Lambda_{N}^{(1)}\left(k, z^{\prime}\right)}{\Lambda_{2}^{(1)}(0,0)}\right] \frac{\omega_{b}\left(k, z^{\prime}\right)}{\omega\left(k, z^{\prime}\right)} \\
&+\frac{\omega(0, z)}{\omega(k, z)}\left(\frac{\omega_{b}(k, z)}{\omega_{b}(0, z)}-1\right) F_{4}(0, z, 0) \Lambda_{N}\left(k, z^{\prime}\right) .
\end{aligned}
$$

The decrease of $R_{N+2}$ follows from that of $R^{(1)}$ and of the decrease in $1 /|z|$ of $\left(\frac{\omega_{b}(k, z)}{\omega_{b}(0, z)}-1\right) \cdot\left[\right.$ Factors $\frac{\omega_{b}(k, z)}{\omega(k, z)}, \frac{\omega(0, z)}{\omega(k, z)}, \ldots \rightarrow 1$ as $\left.z \rightarrow \infty\right]$.

b) Equation (7) might be established from a comparison of the expressions (33), (34) of $\Lambda^{(1)}$ and $R^{(1)}$ and from (42), (4.2'). However, this procedure is somewhat difficult and we outline here an alternative one, based on a complementary analysis made directly, from the outset, on connected functions that are not semiamputated with respect to 1,2 . In this case, the renormalization procedure is analogous to above but the external propagators are now included in the diagrams $\mathbf{D}^{(j)}$ (and have different values at $k=p_{1}+p_{2}$ and at $k=0$. The latter are those occurring in relevant subtracted terms). We here start with finite values of the momentum space cut-off $\varrho$ (left implicit) and will below establish (7) for any given value of $\varrho$. The expression (7) of $\Lambda_{N}$ is then equivalent, in view of (5), to:

$$
\Lambda_{N}\left(k, z^{\prime}\right)=\frac{\delta_{2, N}+\left(1 \omega F_{N+2}\right)\left(k, z^{\prime}\right)}{1+\left(1 \omega F_{4}\right)(0,0)},
$$


where $\varrho$ is again left implicit. Equation (43) can e.g. be derived from (7) by integrating (at $\varrho$ finite) both sides of (5) with the measure $\omega(k, z) d z$. This gives the relation:

$$
\left(1 \omega F_{N+2}\right)\left(k, z^{\prime}\right)=\left(1 \omega F_{4}\right)(0,0) \Lambda_{N}\left(k, z^{\prime}\right)+\left(1 \omega R_{N+2}\right)\left(k, z^{\prime}\right)
$$

which, together with (7), yields (43). When $\varrho \rightarrow \infty$, both $1 \omega F_{N+2}$ and $1 \omega F_{4}$ tend to infinity in view of the behaviour of $F_{N+2}$ and $F_{4}$, so that Eq. (7') will follow at $\varrho$ infinite.

We do not attempt below to make a direct study of convergence properties in the $\varrho \rightarrow \infty$ limit. The (uniform) convergence of the integral $1 \omega R_{N+2}$ [and hence the existence of the $\varrho \rightarrow \infty$ limit in $\left.\left(7^{\prime}\right)\right]$ follows from our previous methods.

Proof of (7) ( $\varrho$ Finite). The analysis is made along the same lines as above, with $\lambda_{1}(z)=\lambda_{\varrho}+\sum_{n=2}^{\varrho} \delta \lambda_{n}(z)$ now replaced by $\mu(z)=\sum_{i=2}^{\varrho} \delta \mu_{n}(z)$. [It is not convenient to include in $\mu(z)$ the analogue of $\lambda_{\varrho}$.] It gives:

$$
S_{N+2}^{c}\left(k, z, z^{\prime}\right)=\chi(z) \hat{\Lambda}_{N}^{(1)}\left(k, z^{\prime}\right) \omega_{b}\left(k, z^{\prime}\right)+\hat{R}_{N+2}^{(1)}\left(k, z, z^{\prime}\right) \omega_{b}\left(k, z^{\prime}\right) .
$$

The link between $\chi(z)$ and $\lambda_{1}(z)$ or $R^{(1)}$ and $\hat{R}^{(1)}$ is not directly transparent, but $\hat{\Lambda}_{N}^{(1)}$ does coincide with $\Lambda_{N}^{(1)}$ as is clear from the comparison of the two procedures:

$$
\hat{\Lambda}_{N}^{(1)}\left(k, z^{\prime}\right) \equiv \Lambda_{N}^{(1)}\left(k, z^{\prime}\right) .
$$

On the other hand, the following relation between $\hat{\Lambda}_{N}^{(1)}$ and $\hat{R}_{N+2}^{(1)}$ holds in view of definitions of $\hat{\Lambda}^{(1)}$ and $\hat{R}^{(1)}$ :

$$
\Lambda_{N}^{(1)} \equiv \hat{\Lambda}_{N}^{(1)}\left(k, z^{\prime}\right)=\frac{\omega\left(k, z^{\prime}\right)}{\omega_{b}\left(k, z^{\prime}\right)} \delta_{2, N}+\int \hat{R}_{N+2}^{(1)}\left(k, z, z^{\prime}\right) d z
$$

The first term in (46) corresponds at $N=2$ to cases in which diagrams are no longer connected when the two lines attached to the same vertex as 1,2 are "separated" from that vertex: this gives the contribution $\omega(k, z)(=$ product of the two 2-point functions) to $\hat{\Lambda}_{2}^{(1)} \omega_{b}\left(k, z^{\prime}\right)$.

From (44), one derives in the same way as (41) was derived from (23) [and using the definition (42) of $\Lambda_{N}$ ]:

$$
S_{N+2}^{c}\left(k, z, z^{\prime}\right)=\frac{S_{4}^{c}(0, z, 0)}{\omega(0,0)} \Lambda_{N}\left(k, z^{\prime}\right) \omega\left(k, z^{\prime}\right)+R_{N+2}^{\prime}\left(k, z, z^{\prime}\right),
$$

where

$$
R_{N+2}^{\prime}\left(k, z, z^{\prime}\right)=\left[\hat{R}_{N+2}^{(1)}\left(k, z, z^{\prime}\right)-\hat{R}_{4}^{(1)}(0, z, 0) \frac{\Lambda_{N}^{(1)}\left(k, z^{\prime}\right)}{\Lambda_{2}^{(1)}(0,0)}\right] \omega_{b}\left(k, z^{\prime}\right) .
$$

A comparison of (48) with (5) shows that:

$$
\omega(k, z) R_{N+2}\left(k, z, z^{\prime}\right) \omega\left(k, z^{\prime}\right)=R_{N+2}^{\prime}\left(k, z, z^{\prime}\right),
$$

and Eq. (7) is then a trivial consequence of (46).

This ends the proof of Theorem 4'. As already announced in Sect. 1, Theorem 4 itself, in which $\varepsilon$ is removed, is a byproduct of the analysis of $R_{N+2}$ given in Sect. 3.2. 
3.2 Second Order (Outline). As in Sect. 3.1, it is convenient to treat semiamputated functions unless recourse is made to "particle analysis" in all slices (allowing us to recover directly results on the amputated functions). We start from (29) and now wish to obtain results on $R^{(1)}$ from the following renormalization procedure, applying to diagrams $\mathbf{D}^{(j)}, j<i(z)$, with 4 outgoing lines below $j$ or an equivalent number of lines and gradients (in power counting): two lines with momenta $k_{1}, k_{2}$ and one gradient acting either on $k=k_{1}+k_{2}$ or on $u=\left(k_{1}-k_{2}\right) / 2$. These three cases will be represented below by indices $\alpha=1,2,3 ; \delta \mu_{j}^{\alpha}(z)$ will denote the sum of the values of diagrams $\mathbf{D}^{(j, \alpha)}$ whose outgoing momenta below $j$ have been fixed at zero and we put:

$$
\mu_{j}^{\alpha}(z)=\sum_{j^{\prime}=j+1}^{i(z)} \delta \mu_{j^{\prime}}^{\alpha}(z)
$$

The analysis then yields a decomposition of $R^{(1)}$ of the form:

$$
R^{(1)}\left(k, z, z^{\prime}\right)=\sum_{\alpha=1}^{3} \sum_{j=2}^{i} \mu_{j}^{\alpha}(z) \Theta^{j, \alpha}\left(k, z^{\prime}\right)+R^{(1)^{\prime}}\left(k, z, z^{\prime}\right),
$$

where $R^{(1)}$ is a sum of diagrams that are renormalized by the subtraction of the $\delta \mu$ 's described above.

Diagrams involved in $\delta \mu_{j}^{\alpha}(z)$ are either diagrams in which 1,2 arrive on two different vertices or diagrams that already start with a vertex with coupling $\mu^{\beta}(z)$ and (besides 1,2) either 4 outgoing lines or 2 outgoing lines and a gradient acting on $\left(k_{1}-k_{2}\right) / 2$ :

$$
\delta \mu_{\alpha}^{j}(z)=\sum_{l \geqq j} \sum_{\beta=1,3} \mu_{l}^{\beta}(z) \Theta_{j, \alpha}^{l, \beta}+K^{j, \alpha}(z),
$$

where $K^{j, \alpha}(z)$ decreases at least like $1 /|z|^{2(i(z)-j)(1-\varepsilon)}$ as $z \rightarrow \infty, \lambda_{\text {ren }}<O(1) \lambda_{0} \varepsilon\left(\lambda_{0}\right.$ small enough).

From (51), (52), one obtains in turn formulae of the form:

$$
R^{(1)}\left(k, z, z^{\prime}\right)=\sum_{\alpha} \mu_{1}^{\alpha}(z) H^{1, \alpha}\left(k, z^{\prime}\right)+R^{(2)}\left(k, z, z^{\prime}\right),
$$

where $H^{1, \alpha}$ and $R^{(2)}$ are shown in the same way as in Sect. 3.1 to be well defined in the limit of infinite momentum cut-off $\varrho$, and $R^{(2)}$ decreases at least like $1 /|z|^{2-\varepsilon}$ as $z \rightarrow \infty, \forall \varepsilon>0, \lambda_{\text {ren }}<O(1) \lambda_{0} \varepsilon$. Formulae defining $H^{1, \alpha}$ and $R^{(2)}$ in terms of the functions $\Theta$ and $K$ are analogous to those of Sect. 3.1. [For $\alpha=3, H^{1,3}$ reduces to $\left.\sum_{j} \Theta^{j, 3}\left(k, z^{\prime}\right) \cdot\right]$

Finally the behaviour of $\mu_{1}^{\alpha}(z)$ as $z \rightarrow \infty$ can also be evaluated as in Sect. 3.1, with here:

$$
\mu_{j}^{\alpha}=\mu_{j+1}^{\alpha}+\delta \mu_{j+1}^{\alpha}=\mu_{j+1}^{\alpha}\left(1+\delta_{\alpha} \beta_{2} \ln M \lambda_{j+1}\right)+\ldots, \quad \delta_{\alpha} \leqq 1,
$$

and hence

$$
\mu_{1}^{\alpha}(z) \approx \mu_{i}^{\alpha}(z) \prod_{j=1}^{i}\left(1+\delta_{\alpha} \beta_{2} \ln M \lambda_{j}\right),
$$

where $i=i(z)$. Noting that $\mu_{i}(z)=\delta \mu_{i}(z)$ contains factors $\lambda_{i}^{2}$ (two couplings at least) 
and $M^{-i}$, one checks that:

$$
\mu_{1}^{\alpha}(z) \approx \frac{1}{|z|(\ln |z|)^{2-\delta_{\alpha}}}
$$

at $z$ large. This result allows one to complete the proof of Theorem 4:

Proof of Theorem 4 (Completed). The results above on $\mu_{1}^{\alpha}$ and $R^{(2)}$ show, in view of (53), that $R^{(1)}$ decreases at least like $1 /|z|$ (and in fact like $1 /|z| \ln |z|$ ). From (43), $R_{N+2}$ decreases itself like $1 /|z|(\ln |z|)^{1-\sigma}$, Q.E.D.

Equation (29) and (53) provide on the other hand an expansion of $H\left(k, z, z^{\prime}\right)$ of the form:

$$
H_{N+2}\left(k, z, z^{\prime}\right)=\lambda_{1}(z) \Lambda_{N}^{(1)}\left(k, z^{\prime}\right)+\sum_{\alpha=1}^{3} \mu_{1}^{\alpha}(z) H_{N}^{1, \alpha}\left(k, z^{\prime}\right)+R^{(2)}\left(k, z, z^{\prime}\right) .
$$

The passage to expressions of $F_{N+2}$ in terms of physical quantities can be made in a way analogous to that presented in Sect. 3.1. Putting:

$$
\begin{aligned}
& \lambda_{\text {ren }}(z)=F_{4}(0, z, 0), \\
& \mu_{\text {ren }}^{(1)}(z)=F_{2 \rightarrow 4}(0, z, 0)-F_{4}(0, z, 0) \Lambda_{4}(0,0), \\
& \mu_{\text {ren }}^{(2)}(z)=\left.\left\{\nabla_{k}\left[F_{2 \rightarrow 2}(k, z, 0)-F_{4}(0, z, 0) \Lambda_{2}(k, 0)\right]\right\}\right|_{k=0}, \\
& \mu_{\text {ren }}^{(3)}(z)=\left.\left\{\nabla_{z^{\prime}}\left[F_{2 \rightarrow 2}\left(0, z, z^{\prime}\right)-F_{4}(0, z, 0) \Lambda_{2}\left(0, z^{\prime}\right)\right]\right\}\right|_{z^{\prime}=0},
\end{aligned}
$$

one gets:

$$
\begin{aligned}
F_{N+2}\left(k, z, z^{\prime}\right)= & \frac{\omega(0, z)}{\omega(k, z)} \lambda_{\mathrm{ren}}(z) \Lambda_{N}\left(k, z^{\prime}\right) \\
& +\sum_{\alpha} \frac{\omega(0, z)}{\omega(k, z)} \mu_{\mathrm{ren}}^{\alpha}(z) H_{N}^{\alpha}\left(k, z^{\prime}\right)+\Sigma_{N+2}\left(k, z, z^{\prime}\right),
\end{aligned}
$$

where $\Sigma_{N+2}$ is shown to decrease at least like $1 /|z|^{2-\varepsilon}, \varepsilon>0$ for $\lambda_{\text {ren }}<O(1) \lambda_{0} \varepsilon$ and $\mu_{\mathrm{ren}}^{\alpha}(z)$ to behave like $1 /|z|(\ln |z|)^{1-\sigma}$ at large $z$. An analysis to third order would allow one to remove restrictions relative to $\varepsilon$ on $\Sigma_{N+2}$.

Remark. As in Sect. 3.1, the result (62) can be directly established in a way that avoids the algebraic problems in the passage from (57) to (62) if "particle analysis" (= cluster expansions of order 4 in all slices) are made from the outset, allowing one to obtain direct expansions of amputated functions as also to define directly the quantities $\lambda_{\text {ren }}(z), \mu_{\text {ren }}^{\alpha}(z)$ by one more step from $\lambda_{1}(z), \mu_{\text {ren }}^{1, \alpha}(z)$.

\section{4. "Non-Euclidean" Results (Outline)}

In this section, we wish to explain how results of Sects. 2 and 3 on the behaviour at large euclidean $z$ can be extended to given non-euclidean, in particular Minkowskian, values of $k, z^{\prime}$. We restrict ourselves here to the region $s<16 \mu^{2}-\varepsilon, \varepsilon>0$, 
where $s$ is the Minkowskian squared c.m. energy, $\mu$ is the physical mass and $\varepsilon$ can be chosen arbitrarily small if $\lambda_{\text {ren }}$ is sufficiently small. (Further results in higher energy regions might be obtained similarly via a more detailed "particle analysis," i.e. the introduction of more general irreducible kernels and structural equations $[9,4,10]$.) For simplicity, we only consider below the 4-point function, but the extension to $(N+2)$-point functions is carried out with only minor changes (by introducing a supplementary $2 \rightarrow N$ 2-particle irreducible kernel). In the region $s<4 \mu^{2}-\varepsilon, \varepsilon>0$ (fixed), all results on $F$ are obtained without change, using the analyticity of $F$ itself in that region (due, by Fourier-Laplace transformation, to exponential fall off properties in euclidean space-time). For the further analysis up to $s=16 \mu^{2}-\varepsilon$, it is convenient to consider the (4-point) Bethe-Salpeter type kernel $\widehat{G}_{M}$ defined in [4] and shown there to be analytic up to $s=16 \mu^{2}-\varepsilon$ and to be linked to $F$ by the regularized B.S. equation, of the type introduced in [11]:

$$
F=\widehat{G}_{M}+\widehat{G}_{M} 0_{M} F
$$

where $0_{M}$ denotes a Feynman-type convolution with modified 2-point functions on the two internal lines: these modified functions have a decrease at infinity in euclidean directions in $e^{-\operatorname{cst} p^{2}}$ so that there will be no ultraviolet problem. The integration contour in $0_{M}$, which is initially euclidean space, is more generally a locally distorted contour $\Gamma(k)$, depending on the energy-momentum $k$, when $k$ goes to the Minkowski region, in order to avoid pole singularities of the 2-point functions [11]. (It still coincides with euclidean space away from a bounded region.)

The Neumann series:

$$
F=\widehat{G}_{M}+\widehat{G}_{M} 0_{M} \widehat{G}_{M}+\ldots
$$

of $F$ is convergent (at small enough renormalized coupling $\lambda_{\text {ren }}$ ) for values of $s$ outside a given neighbourhood $V$ of the 2-particle threshold $s=4 \mu^{2}$ (which can be chosen arbitrarily small if $\lambda_{\text {ren }}$ is sufficiently small).

Large momentum properties of $\hat{G}_{M}$ completely analogous to those of $F$ are established by the same methods as in Sects. 2, 3, in view of its definition. They hold for all values of $k$ up to $s=16 \mu^{2}-\varepsilon ; z$ or $z^{\prime}$ can also be taken away from euclidean space.

The cut-off factors arising from the decrease of modified 2-point functions on internal lines in the definition of $0_{M}$ and the convergence in turn of the series (64) (outside $V$ ) then allow one, by taking apart the factor in $1 / \ln |z|^{1-\sigma}$ in the bounds on the first factor $\hat{G}_{M}$ on the left, to reobtain the analogue of Theorem 2 .

Concerning Wilson expansion, a similar result holds again on $\hat{G}_{M}$ (with different functions $\left.\Lambda_{(M)}^{(1)}, R_{(M)}^{(1)}\right)$ :

$$
\widehat{G}_{M}\left(k, z, z^{\prime}\right)=\lambda_{1}(z) \Lambda_{(M)}^{(1)}\left(k, z^{\prime}\right)+R_{(M)}^{(1)}\left(k, z, z^{\prime}\right),
$$

and in turn, using the expression of $\lambda_{1}(z)$ in terms of $F_{4}(0, z, 0)$ obtained in Sect. 2:

$$
\widehat{G}_{M}\left(k, z, z^{\prime}\right)=\frac{\omega(0, z)}{\omega(k, z)} F_{4}(0, z, 0) \Lambda_{(M)}^{\prime}+R_{(M)}^{\prime}\left(k, z, z^{\prime}\right)
$$


with again a decrease of $R_{(M)}^{\prime}$ in $1 /|z|$ as $|z| \rightarrow \infty$ in euclidean space, $k, z^{\prime}$ fixed. On the other hand, taking into account the behaviour with respect to $z^{\prime}$ yields at most supplementary powers of $|\zeta|$ in bounds on $\Lambda_{(M)}^{\prime}(k, \zeta)$ and $R_{(M)}^{\prime}(k, z, \zeta)$ [where $\zeta$ denotes the integration variable in the convolution of $\Lambda_{(M)}^{\prime}$ or $R_{(M)}^{\prime}$ with the next kernel $\hat{G}_{M}$ in (64)]. In view of the cut-off factors in $e^{-\operatorname{cst} \zeta^{2}}$ in $0_{M}$, Eq. (64) thus yields factorization properties of the form (5) with suitable functions $\Lambda_{N}, R_{N+2}, R_{N+2}$ satisfying bounds of the form (6). In view of the unicity of the terms $\Lambda_{N}, R_{N+2}$ whenever $R_{N+2}$ satisfies such bounds (see remark below Lemma 2 in Sect. 3), the functions $\Lambda_{N}, R_{N+2}$ thus obtained do coincide with those obtained previously when $s<4 \mu^{2}$ and thus appear as analytic continuations of the previous ones for values of $s$ up to $16 \mu^{2}-\varepsilon$. In particular (7) also follows by analytic continuation.

Higher order expansions of $F$ in the region $s<16 \mu^{2}-\varepsilon$ follow similarly.

We finally come back to the neighborhood of $s=4 \mu^{2}$ previously excluded where the sum (64) is no longer necessarily convergent in view of kinematical factors. Following [12], it is convenient to introduce the kernel $U$ defined in terms of $\hat{G}_{M}$ by the equation:

$$
U=\widehat{G}_{M}+U \nabla_{M} \widehat{G}_{M},
$$

where $\nabla=0_{M}-\frac{1}{2} *$ and $*$ denotes on-mass-shell convolution. In contrast to $0_{M}, \nabla_{M}$ is completely regular in $V$. From (67), $U$ is then shown to be locally analytic as $\hat{G}_{M}$ and satisfies analogous large momentum and short-distance properties, such as (66). On the other hand, $F$ is given in terms of $U$ by the formula:

$$
F=U+\frac{1}{2} U * F=U+U_{\mid}(k, z) B(s)_{\mid} U\left(k, z^{\prime}\right),
$$

where $U \equiv U\left(k, z, z^{\prime}\right), U_{1}$ is the restriction of $U$ to values of $z^{\prime}$ such that $\left(\frac{k}{2} \pm z^{\prime}\right)^{2}$ $+\mu^{2}=0$ (mass shell conditions on the right), $U$ is defined similarly and $B$ is a matrix depending only on $s$ (see details in [12]).

The Wilson expansion of $F$ then follows from that of $U$.

Acknowledgements. We are pleased to thank R. Sénéor for several discussions, and C. de Calan for careful reading of the manuscript.

\section{References}

1. Mitter, P., Weisz, P.H.: Phys. Rev. D 8, 4410 (1973)

Gross, D., Neveu, A.: Phys. Rev. D 10, 3235 (1974)

2. Feldman, J., Magnen, J., Rivasseau, V., Sénéor, R.: Commun. Math. Phys. 103, 67 (1986)

3. Gawedzski, K., Kupiainen, A.: Commun. Math. Phys. 99, 197 (1985)

4. Iagolnitzer, D., Magnen, J.: Commun. Math. Phys. 111, 81 (1987)

5. Wilson, K.: Phys. Rev. 179, 1499 (1969)

6. Zimmerman, W.: Ann. Phys. 77, 536 (1973)

7. a) Symanzik, K.: Commun. Math. Phys. 23, 49 (1971), 34, 7 (1973)

b) Bros, J., Ducomet, B.: Ann. Inst. H. Poincaré 45, 173 (1986)

8. a) Callan, C.G.: Methods in field theory. In: Balian, R., Zinn-Justin, J. (eds.). Amsterdam: North-Holland 1976, p. 41

b) Gross, D.J.: Methods in field theory, opcit, p. 141 
9. Iagolnitzer, D., Magnen, J.: Commun. Math. Phys. 110, 51 (1987)

10. Iagolnitzer, D.: VIII ${ }^{\text {th }}$ International Congress on Mathematical Physics. Mebkbout, M., Sénéor, R. (eds.). Singapore: World Scientific 1987, p. 622

11. Bros, J.: Analytic methods in mathematical physics. New York: Gordon and Breach 1970, p. 85

12. Iagolnitzer, D.: Commun. Math. Phys. 88, 235 (1983);

Bros, J., Iagolnitzer, D.: 2-particle asymptotic completeness and bound states in weakly coupled field theories. Saclay preprint (1988)

Communicated by K. Gawedzki

Received April 10, 1988 